\title{
Using Bioinformatics to Identify Novel Druggable Targets for Retinal Neovascularisation Causing Loss of Vision: Transforming Growth Factor Beta- Activated Kinase 1 (TAK1)
}

\author{
Greg J Dusting ${ }^{1}$, Jiang-Hui Wang ${ }^{1,2}$, Damien Ling ${ }^{2,3}$, Leilei $\mathrm{Tu}^{2,4}$, Moeen Riaz ${ }^{1,2}$, Peter Van \\ Wijngaarden $^{1,2}$, Guei-Sheung Liu ${ }^{1,2,5}$ \\ ${ }^{I}$ Ophthalmology, Department of Surgery, University of Melbourne, Australia, ${ }^{2}$ Centre for Eye Research Australia, \\ Australia, ${ }^{3}$ Opthalmology, University of Sydney, Australia, ${ }^{4}$ Ophthalmology, First Affiliated Hospital of Jinan \\ University, Guangzhou, China, ${ }^{5}$ Menzies Institute for Medical Research, University of Tasmania, Hobart, Australia
}

Background: Therapeutic proteins that suppress Vascular Endothelial Growth Factor (VEGF) and inhibit retinal neovascularisation are ineffective in some patients, implicating other factors are involved in retinal neovascularisation that causes loss of vision. Using a rat model of oxygen-induced retinopathy (OIR), we sought to identify novel therapeutic targets for this excessive blood vessel growth using bioinformatics.

Methods: MicroRNA next-generation sequencing was performed with retinal RNA isolated from normoxic controls and rats subjected to OIR. Bioinformatic analysis was undertaken to identify candidate pathways and genes involved in retinal neovascularisation, and candidate genes were validated by RT-qPCR. Transforming Growth Factor beta-Activated Kinase 1 (TAK1) was identified by the intersection of 3 signalling pathways predicted by bioinformatic analysis of key miRNA downregulated in OIR. The selective TAK1 inhibitor, 5Z-7-oxozeaenol (Oxo), was used in angiogenesis assays in vitro to assess endothelial cell proliferation, migration and tube formation on matrix. Oxo and vehicle control were given intravitreally on postnatal day 14 (P14) in OIR rats, when they were returned to room air after intermittent hyperoxia.

Results: TAK1 was identified through bioinformatic analysis as a potential key target and was found to be up-regulated in the retina of OIR rats. Human endothelial cells treated with Oxо (0.01-1 $\mu \mathrm{M})$ showed significant reductions in lumen formation (40.2\% reduction, $\mathrm{p}<0.001)$ and branching (56.2\% reduction, $\mathrm{p}<0.001)$ as well as cell migration in a scratch assay $(63.2 \%$ reduction, $\mathrm{p}<0.001)$ relative to those treated with vehicle alone. Oxo also suppressed vascular sprouting from mouse aortic ring explants $(45.8 \%$ reduction, $\mathrm{p}<0.001)$ relative to those treated with vehicle. In the OIR rats, there was a significant reduction in retinal neovascularisation in eyes that had received either low (18ng) or high (90ng) dose Oxo (50.0\% and 53.3\% reduction, respectively, $\mathrm{p}<0.001$, compared with vehicle, Figure).

Conclusions: Computational analysis of next-generation sequencing data proved useful for screening novel genes modulated in the complex pathology of retinal neovascularisation. The data suggest that TAK1 is involved in development of adverse retinal neovascularisation, and might represent a suitable target for new therapeutics to prevent excessive blood vessel development in diseases such as proliferative diabetic retinopathy. 